\title{
DIVISION IV / WORKING GROUP ABUNDANCES IN RED-GIANTS
}

CHAIR
MEMBERS

\author{
John C. Lattanzio \\ Pavel A. Denissenkov, Roberto Gallino, \\ Josef Hron, Uffe Gråe Jørgensen, \\ Sun Kwok, Jacobus Th. van Loon, \\ Verne V. Smith, Christopher Tout, \\ Robert F. Wing, Ernst K. Zinner
}

\section{TRIENNIAL REPORT 2006 - 2009}

\section{Meetings}

The main activity of the WG on Abundances in Red Giants has been to propose a JD for the IAU GA in 2009. The increasing evidence for distinct populations within globular clusters is leading to the view that there is a continuum between globular clusters and the smallest of the galaxies. Our JD was designed to investigate this link. However, our JD was incorporated into IAU Symposium No. 266 Star Clusters: Basic Building Blocks throughout Time and Space for the IAU XXVII in Rio de Janeiro, 2009. We will be responsible for organising one session in the Symposium to cover the agenda put forward in our JD proposal.

On a similar topic, many researchers associated with the WG will be attending a MaxPlanck Workshop on the similar topic Chemical Evolution of Dwarf Galaxies and Stellar Clusters in July 2008.

Members of the WG were closely involved in the organisation of a Royal Astronomical Society specialist discussion meeting on Super-AGB stars, in London, February 2008. The conclusion of this meeting was that the single biggest problem for these stars is mass-loss, because it is both crucial and unknown.

\section{Newsletter}

The AGB Newsletter, run by Jacco van Loon, a member of the WG, and Albert Zijlstra is to be more formally linked to the WG in future. It will form the main organ of communication with researchers in the field, and is distributed regularly.

\section{Highlights}

Recent scientific highlights of prime interest to our WG include the continuing investigation into mechanisms for driving the 'deep-mixing' known to occur in red-giants and almost certainly AGB stars as well. Such work includes investigations of thermohaline mixing and magnetic effects (Eggleton et al. 2006, 2008; Charbonnel \& Zahn 2007; Busso et al. 2007; Stancliffe et al. 2007). Implications of these mechanisms are still being investigated but are very promising. 
Years ago, Marigo (2002) emphasised the consequences of a carbon-rich envelope and the opacity effects on subsequent thermal pulsing evolution. Recent work by Cristallo et al. (2007) has begun to address this.

Super-AGB stars have received a lot of attention recently. These are stars that ignite $\mathrm{C}$ under degenerate conditions but still go on to experience thermal pulses, and have masses between about 8 and $12 \mathrm{M}_{\odot}$. Important papers are those by Siess $(2006,2007)$ and Poelarends et al. (2008), which investigate the evolution of these stars and emphasise the difficulties of such calculations. We are still awaiting a detailed calculation of yields from these stars, and these may be important for the chemical evolution of globular clusters.

McSaveney et al. (2007) attempted a direct quantitative test of Hot Bottom Burning by comparing models with LMC AGB stars. The basic quantitative predictions of the models were verified, although some uncertainties remain, likely due to uncertainties in the details of the properties of the convective envelope.

Recent work on very low metallicty stars has been prompted by increasingly sophisticated and complete observational studies, and includes theses by H. Lau (Cambridge) and S. Campbell (Monash) as well as Lau et al. (2007, 2008). Indeed, three very active cross-disciplinary areas include these and other works on interpretations of very metalpoor stars, research on the interpretation of the wealth of information present in presolar grains, and the interpretation of new measurement of neutron capture cross sections of astrophysical interest.

The field of red-giant evolution and nucleosynthesis is very active and we imagine the WG will have plenty to do in the next triennium.

John C. Lattanzio chair of the Working Group

\section{References}

Busso, M., Wasserburg, G. J., Nollett, K. M., et al. 2007, ApJ, 671, 802

Charbonnel, C. \& Zahn, J.-P. 2007, A\&A, 476, 29

Cristallo, S., Straniero, O., Lederer, M. T., \& Aringer, B. 2007, ApJ, 667, 489

Eggleton, P. P., Dearborn, D. S. P., \& Lattanzio, J. C. 2006, Science, 314, 1580

Eggleton, P. P., Dearborn, D. S. P., \& Lattanzio, J. C. 2007, ApJ, 677, 581

Lau, H. H. B., Stancliffe, R. J., \& Tout, C. A. 2007, MNRAS, 378, 563

Lau, H. H. B., Stancliffe, R. J., \& Tout, C. A. 2008, MNRAS, 385, 301

Marigo, P. 2002, $A \mathscr{E} A, 387,507$

McSaveney, J. A., Wood, P. R., Scholz, M., et al. 2007, MNRAS, 378, 1089

Poelarends, A. J. T., Herwig, F., Langer, N., \& Heger, A. 2008, ApJ, 675, 614

Siess, L. 2006, $A \mathscr{B} A, 448,717$

Siess, L. 2007, $A \mathscr{E} A, 476,893$

Stancliffe, R. J., Glebbeek, E., Izzard, R. G., \& Pols, O. R. 2007, A $\mathscr{G} A$ (Letters), 464, 57 